\title{
Allocation of effort as a function of payoffs for individual tasks in a multitasking environment
}

\author{
Dongyuan Debbie Wang \\ University of North Florida, Jacksonville, Florida \\ Robert W. Proctor \\ Purdue University, West Lafayette, Indiana \\ AND \\ David F. PICK \\ Purdue University Calumet, Hammond, Indiana
}

\begin{abstract}
SYNWORK1 software allows the examination of how payoffs affect the allocation of effort by people when they perform four different tasks (memory search, arithmetic, visual monitoring, and auditory monitoring). In a previous study (Wang, Proctor, \& Pick, 2007), we showed that participants adopted multitasking strategies allocating relative effort appropriate to payoff differences between the arithmetic and memory tasks, but that they exhibited residual effects of prior payoffs when the payoffs were switched. In the present study, we varied the payoff in two different experiments for only one of these tasks, the memory task in Experiment 1 and the arithmetic task in Experiment 2. Doing this allowed consideration of performance for both the task for which payoff changed explicitly and the other cognitive task, for which the payoff difference was implicit (i.e., relative to the explicit payoff that was manipulated). Although participants adjusted performance on the task for which the payoff explicitly varied, the payoff manipulation had less effect than did the explicit payoff manipulations for both tasks used previously. Also, the change in effort on a task resulting from explicitly increasing its payoff was less than that from decreasing the payoff. SYNWORK1 is a good environment for studying multitasking, but has several limitations that need to be addressed to provide a synthetic work environment that allows investigation of a wider range of theoretically relevant issues.
\end{abstract}

People often have to perform multiple tasks concurrently - a situation commonly referred to as multitasking. Multitasking performance depends in part on the allocation of effort, or attentional resources, to the individual tasks in response to priorities and payoffs that are in effect. The payoff for a particular task can vary and may change across different task contexts. For example, when driving, the maintenance of lane position and following distance are not high priority when there is little traffic on the highway and the weather is good. But, in bad weather or heavier traffic, their priority increases and more attentional resources must be devoted to maintaining lane position and following distance. A longer term shift in priorities can occur in other situations, such as the changes in security measures that occur following a major incident. For example, the priority for checking shoes of airline passengers following the attempted shoe bombing of an aircraft in December 2001 increased greatly. How people adjust to payoff changes for one task of several, and how that priority shift influences the performance of the remaining tasks, are the topics of concern in the present study.
Laboratory studies of multiple-task performance have shown that people can vary the amount of attention devoted to the individual tasks as a function of payoffs or instructions for prioritizing the tasks (Gopher, Weil, \& Siegel, 1989). This is particularly so for tasks that are difficult to perform together because they tend to demand the same processing resources (e.g., the stimuli for both tasks are auditory, as opposed to being auditory for one task and visual for the other) or because their nature requires that they be performed serially (e.g., making a manual response with the right hand for each of two tasks; Wickens \& McCarley, 2008). In such situations, executive control processes involved in coordinating and interleaving the tasks are particularly important. Their importance is even greater when more than two tasks need to be performed, as is often the case in situations encountered in the world.

Although many studies have examined trade-offs in task performance in dual-task settings, relatively few have done so for situations in which more than two tasks need to be performed. Multiple-task performance can be examined in synthetic work environments, one of which is SYNWORK1 (Elsmore, 1994). SYNWORK1 consists of

D. D.Wang, dwang@unf.edu 
four tasks performed concurrently on a computer, with the overarching goal of maximizing the total points earned. The tasks include a math task that requires the addition of pairs of three-digit numbers, a memory task that requires a search of a memory set of letters to determine whether a probe letter is included in the set, a visual monitoring task that involves periodically returning a marker to a center position, and an auditory monitoring task that requires the detection of a high-pitch tone among a stream of lower pitch tones. Points are awarded for correct responses on each task and are subtracted for incorrect responses. The math task is self-paced, in contrast with the memory task, which is presented at a fixed rate. The monitoring tasks both have deadlines for responding, because points are deducted when the visual marker reaches the end of the scale, or if a response is not made to the auditory tone within a certain amount of time after its onset. Proctor, Wang, and Pick (1998) conducted a systematic evaluation of SYNWORK1, examining the influence of many factors (including the number of tasks performed and presentation rates), and showed it to be sensitive to both variables.

In the study of Wang, Proctor, and Pick (2007), the payoffs of the memory and math tasks were manipulated concurrently, in opposition, because these two tasks are the most cognitively demanding. One group of participants received 15 points for each correct memory response and 5 points for each correct math problem in eight sessions on the 1st day, and another group received the opposite payoffs. Performance showed that the participants were sensitive to the payoff differences between the memory and math tasks, making more responses for each task when it received the higher point values. These payoff differences for the memory and math tasks had little influence on performance of the two monitoring tasks. On the 2nd day, half of the participants had the payoffs for the memory and math tasks switched in Sessions 9-12, whereas the other half continued to receive the same payoffs as in Sessions 1-8. Participants adjusted performance of the memory and math tasks in accord with the new payoffs, but showed lingering effects of the payoffs that had been used previously.

Our previous results (Wang et al., 2007) indicated that participants are sensitive to payoff differences in the SYNWORK1 environment and that they adjust the amount of effort devoted to the math and memory tasks in accord with the different payoffs for the two tasks. In that study, both of the payoffs for the two tasks were varied in opposition. However, there are also situations in life in which the payoff for a single task is altered, and that type of situation was of primary concern in the present study. Explicit manipulation of payoff for one task produces an implicit manipulation of payoff for the other tasks. For example, when the payoff for the memory task is high, the payoffs for the other tasks will be relatively lower than when the payoff for the memory task is low.

The possible importance of relative payoff in multipletask performance is suggested by the study of concurrent reinforcement schedules. Catania (1963) showed that when a pigeon's pecks on two keys are reinforced concur- rently by two independent variable-interval schedules, one for each key, the response rate on one key depends in part on the reinforcement rate for the other key. This relation is illustrated by the formula known as Herrnstein's (1961, 1990) matching law, which can be stated as:

$$
\mathrm{R}_{1}=\left[\mathrm{r}_{1} /\left(\mathrm{r}_{1}+\mathrm{r}_{2}\right)\right] *\left(\mathrm{R}_{1}+\mathrm{R}_{2}\right),
$$

where $\mathrm{R}$ is the rate of response, $\mathrm{r}$ is the rate of reinforcement, and the subscripts 1 and 2 indicate Key 1 and Key 2. The point of this equation is that the response rate on a task is a function of both the payoff for that task and the payoff for the other task. The matching law has been found to hold for humans as well in some situations (see, e.g., Logue, Forzano, \& Tobin, 1992). If participants are sensitive to the relative payoffs in the SYNWORK1 environment, they should alter performance on the other tasks in accordance with the implicit payoff difference that occurs when the explicit payoff for one task is varied.

An example of an everyday activity in which an increase in payoff for one task may influence the relative priority of another task is that of conversing with another person as a passenger or by cell phone while driving. When the cost of not devoting enough attention to braking the vehicle in response to an event becomes high (i.e., a likely collision), then the priority for conversation should decrease to a very low level. Levy and Pashler (2008) tested the extent to which drivers could cease performing a secondary task when its priority became very low using a dual-task procedure with a driving simulator. The primary driving task required a braking response to the onset of brake lights on a lead vehicle. The secondary task required a choice response indicating whether a stimulus tone had been presented either just once or twice in rapid succession. The presentation of the brake lights was coordinated with the presentation of the tones using three different sequences: a brake-light onset of $150 \mathrm{msec}$ before tones, a tone onset of $150 \mathrm{msec}$ before brake lights, or the simultaneous onset of brake lights and tones. Participants were instructed to ignore the choice task whenever they saw brake lights. However, even with maximum priority assigned to the braking response, and encouragement given to ignore the choice response when braking was required, participants still most often performed the choice response before the braking response, even when onset of the brake lights preceded that of the tone (the tone response was first on $51.3 \%, 63.1 \%$, and $79.1 \%$ of trials when the brake light onset occurred before, simultaneous with, or after the tone, respectively). Thus, participants did not seem to perform in accordance with the priority changes and continued to give the secondary choice task higher priority than warranted when the brake lights came on first.

Because the SYNWORK1 environment consists of four tasks for which performance must be interleaved, only two of which are time constrained, it is possible that people will alter the amount of effort they will devote to tasks when the implicit payoffs change relative to that of a task for which the payoff changes explicitly. On the basis of Wang et al.'s (2007) findings, we expect that when the payoff for a single task is varied, participants should sys- 
tematically alter their performance of that task: If the payoff is greater in one condition than in another, then the task should be performed more frequently in the condition with the higher payoff. When the payoff for that task is high, the relative payoff for the other tasks is lower, and vice versa. If participants are sensitive to the implicit payoff for the tasks in which the explicit payoff is held constant, the performance of those tasks for which the payoff is not directly manipulated should vary in the opposite manner of the task for which the payoff is directly manipulated. Specifically, because the memory and math tasks are both cognitively demanding, a higher payoff for one would lead to a decreased response rate for the other.

Thus, the purpose of the present study was to examine changes in performance as a function of the explicit manipulation of payoffs for one task and the implicit manipulation for the other tasks, particularly that of the other cognitive task. In Experiment 1, the payoff of the memory task was manipulated, whereas in Experiment 2, the payoff of the math task was manipulated. The 1st day of each experiment was designed to examine the sensitivity of participants to the initial payoff differences on the task for which payoff was directly manipulated, as well as any effect that these differences might have on the responses for the other tasks - especially the other cognitive task. The 2 nd day was designed to examine how allocation strategies changed by measuring the effort change on all tasks under transfer conditions in which the payoff was changed only for the task for which there were initial payoff differences (the memory task in Experiment 1 and the math task in Experiment 2).

\section{EXPERIMENT 1}

All four tasks were performed in Experiment 1, with only the memory payoff manipulated, allowing us to examine how performance on the other three tasks changed. We hypothesized that on the 1st day, the memory task should be performed more often when the payoff is high than when it is low. Performance on the other tasksparticularly on the other cognitive task, the math task, should be less when the memory payoff is high if participants are allocating a larger percentage of their total resources to the memory task under the higher payoff.

For the 2nd day, the memory payoff was changed for some participants but not for others. Of interest was whether the effect of increasing the relative payoff for a particular task, which we call positive incentive, is of a magnitude similar to the effect of decreasing the relative payoff, which we call negative incentive. Explicit effects of payoff refer to changes in responses for the memory task, whose payoff was changed directly, whereas implicit effects refer to changes in responses for the other tasks. For explicit effects, positive and negative incentives refer to increases and decreases, respectively, of that payoff in the transfer session; for implicit effects, positive incentive is when the relative payoff for the other tasks increases because the memory payoff decreases, and negative incentive is when the relative payoff for the other tasks decreases because the memory payoff increases.

\section{Method}

\section{Participants}

Sixty students enrolled in introductory psychology classes at Purdue University participated for credit toward a course requirement. This was a 2-day experiment, with each participant performing eight 5 -min sessions on the 1st day and four 5-min sessions on the 2nd day. ${ }^{1}$ Four groups of 15 participants who were defined by 1 st-day payoff and 2nd-day payoff participated in each test. All participants were tested during the same 4-week period, and participants were assigned randomly to one of the four groups.

\section{Apparatus}

The experiment was conducted on personal computers using the SYNWORK1 (Version 2.15) software with an interface program that presented instructions. The display, in which each task occupies one quadrant, or window (see Figure 1), was shown on a 14-in. VGA color monitor. Koss TM01 headphones were used to present the tone stimuli, and responses were made with a Microsoft mouse operated by the right hand. The mouse controlled a cursor that could be positioned on response boxes on the screen, and a response was recorded when the left button of the mouse was clicked with the index finger.

Upper left window: Memory task. In each session, a list of five letters was chosen from the alphabet (excluding the letters $C$, $\mathrm{D}, \mathrm{M}, \mathrm{Q}$, and $\mathrm{V}$ ) and was displayed in a box at the top of the window for $5 \mathrm{sec}$. After that, it was replaced by the words "retrieve list." When this message was displayed, clicking the mouse on the list box resulted in the display of the list for another $5 \mathrm{sec}$, and points were charged for this retrieval. Probe letters were displayed in the box in the center of the window at a 5 -sec rate, with the probe removed when a response was made or after $5 \mathrm{sec}$ had elapsed. The participant's task was to indicate whether or not the probe was a member of the list just displayed by clicking the mouse on either the "Yes" or "No" box at the bottom of the window before the probe disappeared from the screen. The payoff was 15 or 5 points for a correct response, depending on the group, and 5 points were subtracted from the total points for each incorrect response, including failure to respond to the probe.

Upper right window: Math task. Two randomly selected threedigit numbers were presented. The task was to add them together, adjusting the answer by clicking on "+" and "-" boxes located below each character of the answer that was initially set to " 0000 ." Clicking on the "Done" box at the bottom of the window resulted in the pre-

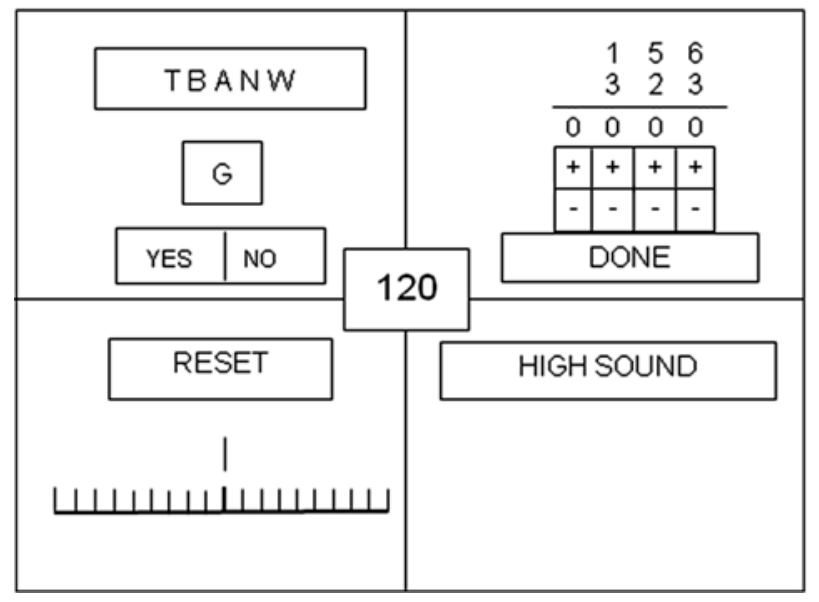

Figure 1. Depiction of display for SYNWORK1. Total points are shown in the centered box. The memory task is in the upper left quadrant, the math task in the upper right quadrant, the visual monitoring task in the lower left quadrant, and the auditory monitoring task in the lower right quadrant. 
sentation of a new problem, as well as the addition of 10 points if the answer was correct, and subtraction of 5 points if it was incorrect. There was no time limit for the completion of each problem, and when a problem was completed, another problem was presented.

Lower left window: Visual monitoring task. A white marker moved from the center of a 201-pixel scale, toward either end, at a rate of 10 pixels $/ \mathrm{sec}$. Clicking the mouse on a box labeled "Reset" at the top of the window reset the marker to the center of the scale. The marker reached one end after $10 \mathrm{sec}$ if the participant did not respond. Points were awarded for each reset according to how far the marker was from the center. The maximum payoff was 10 points. If the marker reached the end of the scale, 2 points were subtracted from the total for every second it remained there. The task was to prevent the pointer from reaching the end of the scale, while scoring the most points.

Lower right window: Auditory monitoring task. Tones of brief duration were presented at a $2.5-\mathrm{sec}$ rate. The tones were of low $(1046 \mathrm{~Hz})$ or of high $(1319 \mathrm{~Hz})$ pitch. The low-pitch tone occurred $80 \%$ of the time and the high-pitch tone $20 \%$ of the time. The order of these tones was randomly determined. The task was to click the "High Sound Report" box in the window during the interval between the occurrence of a high-pitch tone and the next tone in the sequence. The payoff was 10 points for correct answers, and 10 points were subtracted for incorrect answers.

\section{Procedure}

At the beginning of the experiment, the participants were told that they would be performing four tasks concurrently for which they were to receive points, and that their goal was to maximize the amount of points they obtained in each 5-min session. A running total of points obtained was available in the center box on the screen throughout each session as feedback, although no additional feedback about performance in prior sessions or of other subjects was provided. Payoff was only in terms of points toward the task goal of maximizing total points, and no monetary reward was involved. They were then shown a printout of the screen with the four individual tasks (see Figure 1). The experimenter went through each of the four tasks that would be performed and explained verbally the points that would be gained for each correct response and lost for each incorrect response. On that day, two groups differed in the payoff for each correct answer in the memory task, which was 15 or 5 points, whereas the payoff for a correct answer in the math task was held constant at 10 points. The two groups are denoted as memory-15 and memory-5.

On the 2nd day, participants were instructed that they would be performing the same four tasks as on the 1st day. The payoffs in effect for the 2nd day were described verbally, and the experimenter reemphasized that the goal was to maximize the total points obtained in each session. For half of the participants in each group, the payoff of the memory task was switched from that of the initial session. If the initial memory payoff was 15 , the payoff for the transfer session was 5 (memory-15/MEMORY-5). If the initial memory payoff was 5 , the payoff of transfer session was 15 (memory-5/MEMORY-15). The other half of the participants performed under the same payoff in the transfer session as in the initial session.

The parameters were set as follows: For the memory task, the memory set was five letters; the presentation time was $5 \mathrm{sec}$; the interval between stimuli was $5 \mathrm{sec}$; the payoff for correct responses was 15 or 5 points, depending on the group. For each incorrect response, 5 points were subtracted. For the math task, the stimuli were two 3-digit numbers. For each correct answer, the payoff was 10 points; for each incorrect answer, 10 points were subtracted. For the visual and auditory tasks, the parameters were as those described above

\section{Results}

On Day 1, half of the participants received one payoff condition and the other half the other, and on Day 2, each of those groups was split in half to receive the same payoff condition as or different payoff condition than on the 1st day. Consequently, we analyzed Day 1 performance separately from Day 2 performance. ANOVAs were conducted on total points and points for each individual task, with Day 1 payoff as a between-subjects variable and session (1-8) as a within-subjects variable for Day 1 , and with both Day 1 and Day 2 payoffs as between-subjects variables and session (9-12) as a within-subjects variable for Day 2. Additional performance measures were analyzed when necessary.

\section{Day 1}

Total points. The impact of the payoff conditions on the goal performance, total points, is shown in Figure 2. There was a significant Day 1 payoff main effect $\left[F(1,58)=156.09, M S_{\mathrm{e}}=154,440, p<.005\right]$, a session main effect, and a session $\times$ Day 1 payoff interaction $\left[F \mathrm{~s}(7,406)=157.56\right.$ and $\left.8.92, p \mathrm{~s}<.005, M S_{\mathrm{e}}=13,493\right]$. The higher memory payoff group obtained more total points than did the lower payoff group, and the difference between the two groups increased across sessions. The total points increased more rapidly for the memory-15 payoff group than for the memory-5 group. This measure is relatively uninformative because fewer points could be obtained from the memory task for the lower payoff group than for the higher one.

Because each correct memory response was worth three times as many points in the memory- 15 condition as in the memory- 5 condition, a better indication as to whether overall task performance varied as a function of the different memory payoffs is to divide the points for the memory task for each subject in the memory-15 condition by 3 . A new point total was determined for subjects in that condition after adjusting for the point differential

\section{Total Points}

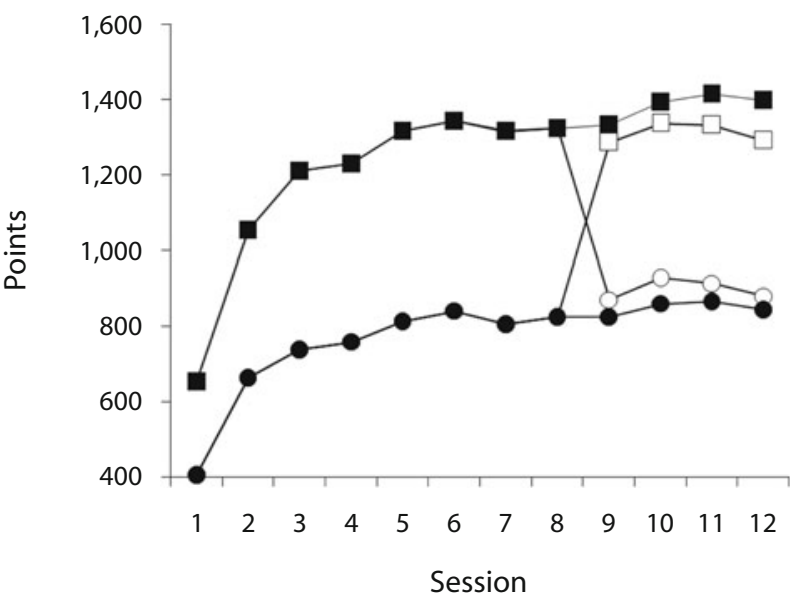

Figure 2. Total points for the different payoff conditions as a function of Sessions 1-8 (1st day) and Sessions 9-12 (2nd day) for Experiment 1. A memory payoff of 15 is indicated by squares, and a memory payoff of $\mathbf{5}$ is indicated by circles; filled symbols for the 2nd day designate continued payoff, and unfilled symbols designate a changed payoff. 
Memory Task

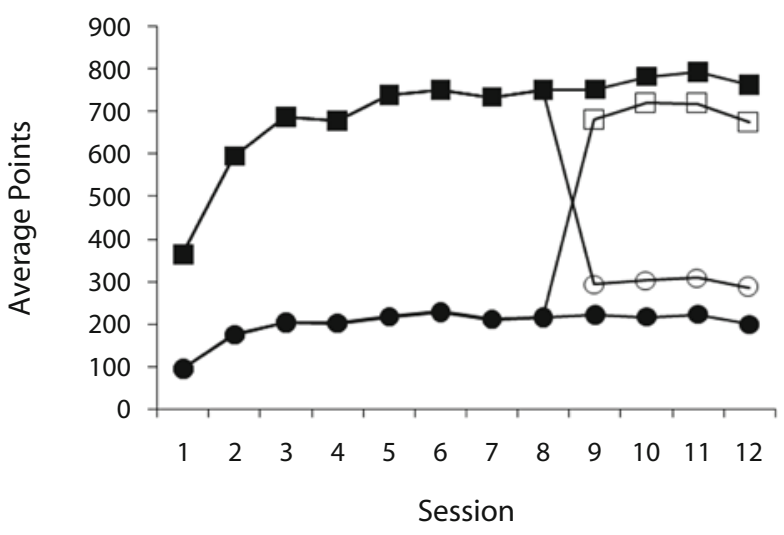

Visual Task

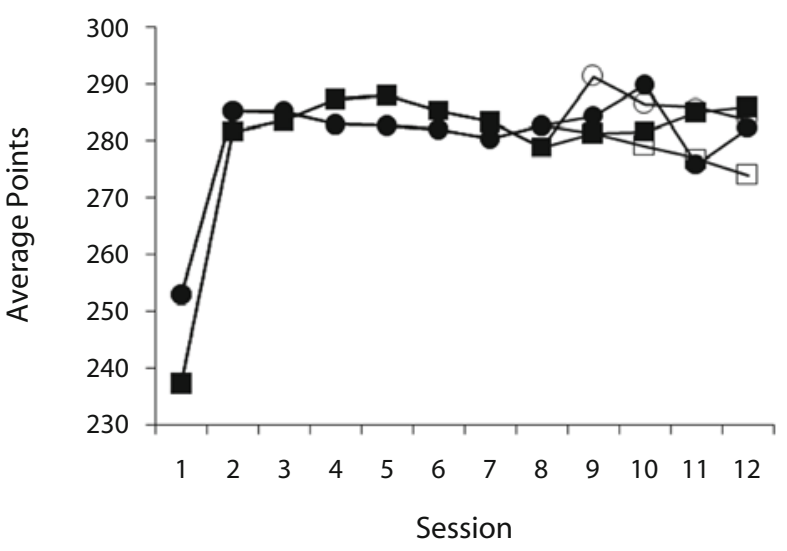

Math Task

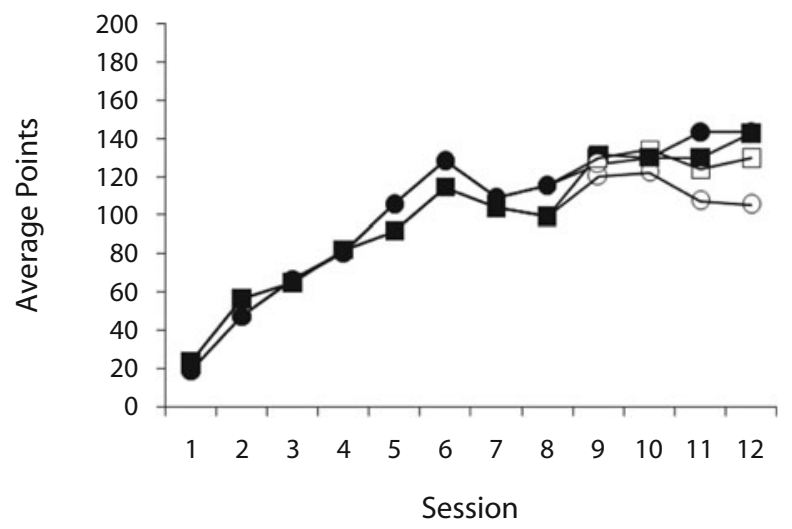

Auditory Task

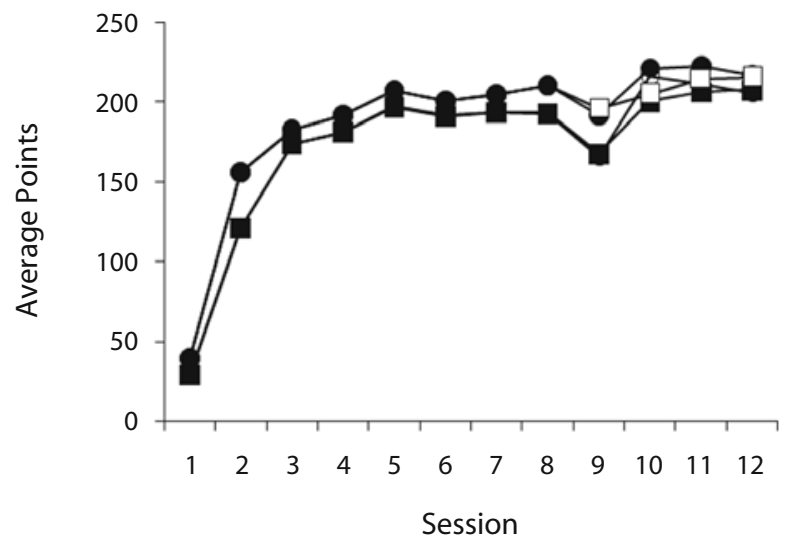

Figure 3. Individual task scores as a function of session and group for Experiment 1 . A memory payoff of 15 is indicated by squares, and a memory payoff of 5 is indicated by circles; filled symbols for the 2 nd day designate continued payoff, and unfilled symbols designate a changed payoff.

in this manner. When adjusted to a common point scale, the only significant effect was that of session $[F(7,406)=$ $\left.146.44, p<.005, M S_{\mathrm{e}}=8,553\right]$. Overall performance for both groups increased similarly across sessions. Thus, the only significant influence of the greater memory payoff on total points was through each correct memory response being worth more points.

Individual tasks. Next, we will discuss the results for the memory and remaining tasks.

Memory. The memory points showed a significant Day 1 payoff main effect $[F(1,58)=312.78, p<.005$, $\left.M S_{\mathrm{e}}=84,106\right]$. They also showed a significant session effect and session $\times$ Day 1 payoff interaction $[F \mathrm{~s}(7,406)=$ 57.93 and $\left.15.02, p \mathrm{~s}<.005, M S_{\mathrm{e}}=7,875\right]$. These results indicate that the difference in memory points between the memory- 15 and memory- 5 conditions increased with practice (see Figure 3, upper left panel).

When the points for the memory-15 group were divided by 3 (for the aforementioned reason), the resulting analysis yielded a Day 1 payoff main effect that approached the .05 level $\left[F(1,58)=3.57, p=.064, M S_{\mathrm{e}}=24,553\right]$. Thus, when adjusted for the point differential, the memory-15 group $(M=221)$ still tended to show an advantage over the memory-5 group $(M=194)$, indicating that they were performing the memory task somewhat more. Also, the adjusted analysis showed a session main effect $[F(7,406)=$ $\left.60.59, p<.005, M S_{\mathrm{e}}=1,865\right]$, showing that both groups increased performance of the memory task across sessions.

The number of memory responses provides another indicator of the effects of payoff. The statistical analysis did not show a significant Day 1 payoff main effect for number of memory responses $(F<1.0)$, but it did show a significant session main effect and a session $\times$ Day 1 payoff interaction (see Figure 4$)\left[F_{\mathrm{S}}(7,406)=37.60\right.$ and $\left.3.55, p \mathrm{~s}<.001, M S_{\mathrm{e}}=33.794\right]$. The number of memory responses increased across sessions, with this increase being larger for the participants who received the high payoff than for those who received the low payoff. Across Sessions 3-8, the high-payoff group made 4.2 more memory responses on average than did the low-payoff group. Thus, the two analyses indicate an effect of the explicit payoff difference on memory task performance.

Remaining tasks. The math points (see Figure 3, upper right panel) showed only a session main effect $[F(7,406)=$ 


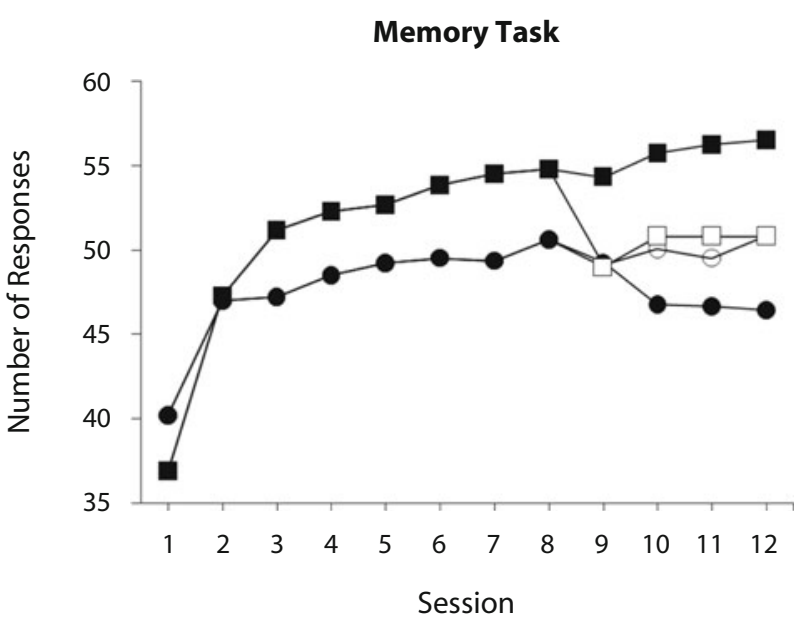

Figure 4. The number of memory responses as a function of Sessions 1-8 (1st day) and Sessions 9-12 (2nd day) for Experiment 1 . A memory payoff of 15 is indicated by squares and a memory payoff of 5 is indicated by circles; filled symbols for the 2nd day designate continued payoff, and unfilled symbols designate a changed payoff.

$\left.59.21, p<.005, M S_{\mathrm{e}}=1,153\right]$. The number of math responses showed no significant payoff main effect or interaction, only a significant session effect (see Figure 5) $\left[F(7,406)=116.96, p<.005, M S_{\mathrm{e}}=8.147\right]$, with the number of math responses increasing across sessions.

The visual and auditory monitoring tasks (see Figure 3, lower panels) also showed only session main effects $\left[F \mathrm{~s}(7,406)=4.317\right.$ and $66.549, p \mathrm{~s}<.005, M S_{\mathrm{e}}=2,602$ and 3,014]. More points were obtained in later sessions than in earlier sessions, with the primary increase for the visual task being between Sessions 1 and 2. Thus, the implicit change in payoffs for the math and monitoring tasks relative to the memory task payoff had no reliable influence on performance.

\section{Day 2}

Total points. The analysis indicated main effects of Day 2 payoff (see Figure 2 ) $[F(1,56)=139.49, p<.005$, $\left.M S_{\mathrm{e}}=96,871\right]$ and session $[F(3,168)=5.25, p<.005$, $\left.M S_{\mathrm{e}}=7,213\right]$. The groups that received the higher memory payoff on Day 2 obtained more points than did the groups that received the lower payoff. An analysis with the memory points for the memory-15 group that was adjusted to account for the higher payoff indicated only a significant session main effect $[F(3,168)=4.45, p=$ $\left..005, M S_{\mathrm{e}}=4,763\right]$.

Individual tasks. Next, we will discuss the results for the memory and remaining tasks.

Memory. The memory score showed significant Day 1 and Day 2 payoff main effects (see Figure 3 ) $[F \mathrm{~s}(1,56)=$ 5.26 and $195.83, p \mathrm{~s}<.026$ and $\left..005, M S_{\mathrm{e}}=69,809\right]$. There was also a significant session effect $[F(3,168)=4.96, p=$ $\left..003, M S_{\mathrm{e}}=2,616\right]$. The groups with the memory-15 payoff on the 2nd day obtained more points than did the groups with the memory-5 payoff, as would be expected, considering the amount of points that could be obtained. More important, as was indicated by the significant Day 1 payoff main effect, the groups who had received the memory-15 payoff on the 1st day obtained more points than did the groups who had received the memory-5 payoff. Participants who had initially received the higher payoff continued to respond more on the memory task on the 2nd day, regardless of whether that payoff was maintained or reduced.

The analysis of adjusted memory points confirmed this conclusion. This analysis showed a session main effect $[F(3,168)=4.17, p=.007]$ and, more importantly, a Day 1 payoff main effect $[F(1,56)=5.26, p=.026](M=$ 224 for memory- 5 and $M=277$ for memory- 15 for the 1 st day). That the main effect of Day 1 payoff remained evident is not surprising, given that this comparison is orthogonal to whether the participants received 5 or 15 points for the memory task on Day 2. The number of memory responses did not show significant Day 1 or Day 2 payoff main effects, or an interaction $\left[F_{\mathrm{S}}(1,56)=1.79,2.22\right.$, and 0.22 , $p \mathrm{~s}=.19, .14$, and $\left..64, M S_{\mathrm{e}}=531\right]$. However, it is apparent in Figure 4 that, consistent with the analysis of memory points, participants who received the higher memory payoff on Day 1 tended to make more memory responses than did participants who received the lower payoff on Day 1. The groups who received the higher payoff on the Day 2 also tended to make more memory responses.

Remaining tasks. The math points showed no significant effect of any of the variables. For the visual monitoring task, no effects were significant either. For the auditory monitoring task, there was a session main effect on score $\left[F(3,168)=17.01, p<.005, M S_{\mathrm{e}}=899\right]$ and on positive tone detection $\left[F(3,168)=24.41, p<.005, M S_{\mathrm{e}}=1.962\right]$. The major trend for these effects was for performance to increase from Sessions 9 to 10 and then to level off, as indicated by the fact that the session effect was not significant $(F<1.0)$ when only Sessions $10-12$ were analyzed.

Positive and negative incentives. We further examined performance in Sessions 9-12 as a function of posi-

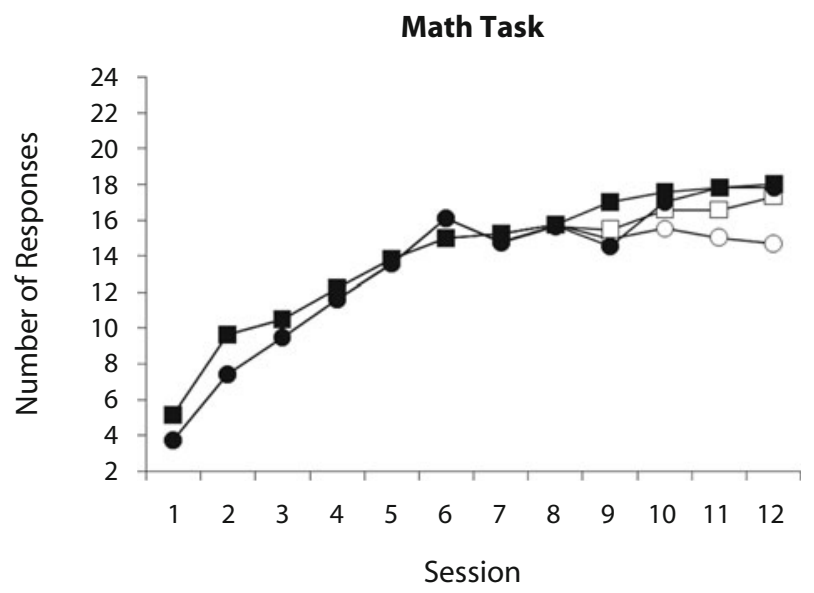

Figure 5. The number of math responses as a function of Sessions 1-8 (1st day) and Sessions 9-12 (2nd day) for Experiment 1. A memory payoff of 15 is indicated by squares, and a memory payoff of 5 is indicated by circles; filled symbols for the 2 nd day designate continued payoff, and unfilled symbols designate a changed payoff. 
tive and negative incentive (i.e., an increase or decrease in relative payoff). This comparison was performed separately for explicit payoff changes (the memory task) and implicit payoff changes (the math task).

Explicit effects. The positive incentive was the performance change when the payoff of the memory task was increased. This result is illustrated by the difference between the memory-5/MEMORY-15 group and memory-5/MEMORY-5 group in the 2nd day, which is 3.05 responses (see Figure 4). However, this difference did not approach statistical significance $[F(1,28)<1.0]$ and was due mainly to a single participant in the memory-5/ MEMORY-5 group who quit performing the memory task.

The negative incentive, on the other hand, was the performance change when the memory payoff was decreased. This result is illustrated by the difference between the memory-15/MEMORY-15 and memory-15/MEMORY-5 groups in Day 2, which was 5.82 responses (see Figure 4). The negative incentive effect also did not achieve the .05 significance level, although it showed a trend toward it $\left[F(1,28)=2.90, p=.10, M S_{\mathrm{e}}=350\right]$. Thus, the data suggest that negative incentive may act to reduce responding on the memory task, but positive incentive seems to have little effect on performance.

Implicit effects. The negative incentive measured the difference between the mean numbers of math responses of the memory-5/MEMORY-5 and memory-5/MEMORY-15 groups. This difference of 0.28 responses was nonsignificant $(F<1)$, showing little indication of an implicit negative incentive effect (see Figure 5). The positive incentive was the difference between the mean of memory-15/ MEMORY-15 and memory-15/MEMORY-5. When the payoff for the memory task is decreased, math performance should increase. To get more points, participants in the memory-15/MEMORY-5 group should have done more math problems than participants in the memory-15/ MEMORY-15 group. But the number of math responses of the memory-15/MEMORY-5 group was 2.5 less than that of the memory-15/MEMORY-15 group $[F(1,28)=1.10$, $\left.M S_{\mathrm{e}}=177.7, p=.30\right]$, being in the opposite direction (see Figure 5). This outcome may be due to the different characteristics of the math and memory tasks. The math task is optional and requires more effort to perform: The sum must be calculated and the appropriate buttons clicked, while not allowing these activities to disrupt performance of the experiment-paced tasks. The memory task is quite different. It requires only a single decision and a single click for a correct response.

\section{Discussion}

For Day 1 , there was a significant session $\times$ Day 1 payoff interaction for the number of memory responses. With practice, participants who had the higher memory payoff increased their number of memory responses to perform more than did participants who had the lower payoff. Thus, they were sensitive to the payoff difference and adjusted their strategies to do more memory responses when they were worth more points. The memory payoff had no significant main effect of or interaction with the performance of the other tasks, including the math task. Participants who received the lower memory payoff did not compensate by performing significantly more math problems.

In the transfer Sessions 9-12, there were no significant Day 1 and Day 2 payoff effects on the number of memory responses. However, the number of responses tended to be larger for those who had the higher payoff on the 1st day, and the effect of Day 1 payoff was significant for memory points. There also was a tendency for those who had the higher memory payoff on Day 2 to make more memory responses than those who had the lower payoff. The number of math responses was not affected significantly by Day 1 payoff, Day 2 payoff, or their interaction.

\section{EXPERIMENT 2}

The design of Experiment 2 was similar to that of Experiment 1 , with the major difference being that the math payoff was manipulated instead of the memory payoff. This experiment was designed to evaluate the sensitivity of math task performance to the explicit payoff change, and of the other tasks - particularly the memory task - to the implicit payoff differences as a function of the math payoff.

\section{Method}

Sixty new participants were taken from the same pool as in Experiment 1 . All participants were tested during the same 4-week period, and participants were assigned randomly to one of the four groups. There were two groups for which the initial payoff for each correct answer in the math task was 15 or 5 points, whereas the payoff for each correct answer in the memory task was held constant at 10 points. On the 2 nd day, half of the participants in each group performed with the payoff of the math task switched from that of the 1st day. If the initial math payoff was 15 (math-15), the payoff of the 2 nd day was 5 (math-15/MATH-5). If the initial math payoff was 5 (math-5), the payoff of 2nd day was 15 (math-5/MATH-15). The other half performed with the same payoff structure as on the 1 st day.

The apparatus was the same as that in Experiment 1. The parameters were set as follows: For the memory task, the memory set was five letters; the presentation time was $5 \mathrm{sec}$, and the interval between probes was increased to $10 \mathrm{sec}$ to make the points attainable from it closer to those that could be obtained from the math task. The payoff for a correct response was 10 points, and 10 points were subtracted from the total points for each incorrect response. For the math task, the stimuli were two 3-digit numbers. For correct answers, the payoff was 15 or 5 points, depending on the group and day. For each incorrect answer, 5 points were subtracted from the total points for each incorrect answer. For the visual and auditory monitoring tasks, the parameters were set the same as in Experiment 1.

\section{Results}

The data were analyzed in a manner similar to that for Experiment 1. The results for Day 1 resembled those of Experiment 1, whereas those for Day 2 deviated in some respects.

\section{Day 1}

Total points. As in Experiment 1, there was a significant Day 1 payoff main effect (see Figure 6) $[F(1,58)=$ $\left.11.17, p=.001, M S_{\mathrm{e}}=111,086\right]$. The session main effect 


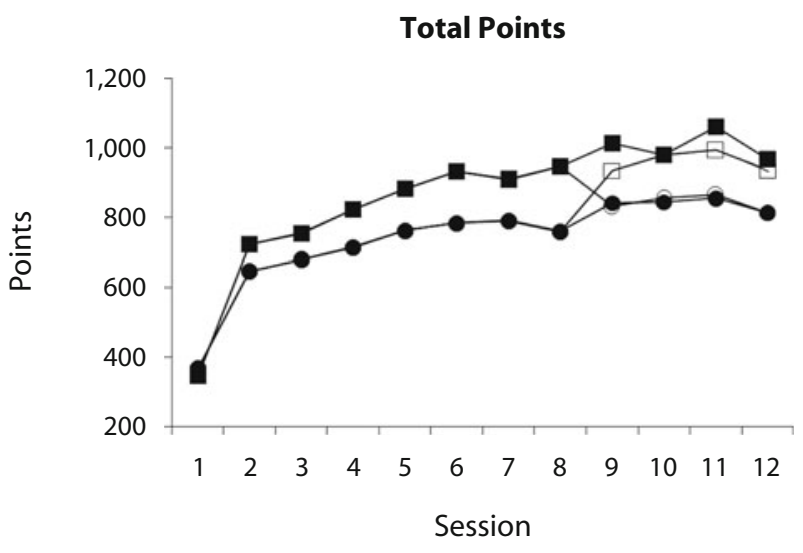

Figure 6. Total points for the different payoff conditions as a function of Sessions 1-8 (1st day) and Sessions 9-12 (2nd day) for Experiment 2. A math payoff of 15 is indicated by squares and a math payoff of 5 is indicated by circles; filled symbols for the 2 nd day designate continued payoff, and unfilled symbols designate a changed payoff.

and session $\times$ Day 1 payoff interaction were significant as well $\left[F_{\mathrm{S}}(7,406)=148.28\right.$ and $5.13, p \mathrm{~s}<.005, M S_{\mathrm{e}}=$ $11,350]$. The math-15 group obtained a higher score than did the math-5 payoff group, and the difference between the groups increased with session. This difference is due at least in part to each math response being worth more points for one group than for the other.

Another analysis was performed as in Experiment 1, with the math points for the math- 15 group divided by 3 to take into account the differential in points received for each correct response. As in the earlier experiment, this analysis showed only a significant session main effect $\left[F(7,406)=125.05, p<.005, M S_{\mathrm{e}}=10,619\right]$.

Individual tasks. Next, we will discuss the results for the math and remaining tasks.

Math task. The math score showed a significant Day 1 payoff effect $\left[F(1,58)=60.02, p<.005, M S_{\mathrm{e}}=35,324\right]$, as well as a session main effect and a session $\times$ Day 1 payoff interaction (see Figure 7, top right panel) $\left[F_{\mathrm{s}}(7,406)=\right.$ 63.04 and 29.34, $\left.p \mathrm{~s}<.005, M S_{\mathrm{e}}=1,218\right]$. The math-15 payoff group obtained more points than did the math-5 payoff group.

As in Experiment 1, the math scores were divided by 3 to adjust for each correct response being worth three times as many points with the math- 15 payoff than with the math-5 payoff. The adjusted math scores showed an effect of Day 1 payoff that approached significance $[F(1,58)=$ $\left.3.91, p=.053, M S_{\mathrm{e}}=6,281\right](M=44.9$ for math-5; $M=$ 55.2 for math-15). They also showed a significant session effect and an interaction with Day 1 payoff $[F(7,406)=$ 47.5 and $\left.4.31, p \mathrm{~s}<.005, M S_{\mathrm{e}}=323\right]$, indicating that math points increased faster with the higher payoff condition than with the lower payoff condition. The number of math responses similarly did not show a significant Day 1 payoff main effect (see Figure 8), but did show a session main effect and a session $\times$ Day 1 payoff interaction $\left[F(7,406)=81.25\right.$ and $2.69, p<.001$ and $.01, M S_{\mathrm{e}}=$ 10.781]. The number of math responses performed across sessions increased more when the payoff was higher than when it was lower.

Remaining tasks. The memory score showed only a significant session main effect (see Figure 7, top left panel) $\left[F(7,406)=44.16, p<.005, M S_{\mathrm{e}}=3,671\right]$, as did the number of memory responses (see Figure 9) $[F(7,406)=$ $\left.35.90, p<.001, M S_{\mathrm{e}}=25.826\right]$.

For the visual monitoring task, the data showed a significant session main effect on task score $[F(7,406)=$ $\left.6.40, p<.005, M S_{\mathrm{e}}=1,255\right]$, resets $[F(7,406)=3.606$, $\left.p=.001, M S_{\mathrm{e}}=108.213\right]$, average distance from center $\left[F(7,406)=2.74, p=.009, M S_{\mathrm{e}}=88.673\right]$, and lapses $\left[F(7,406)=2.6, p=.036, M S_{\mathrm{e}}=1.25\right]$. The auditory monitoring task showed a session main effect on task score $\left[F(7,406)=69.31, p<.005, M S_{\mathrm{e}}=4,437\right]$, positive tone detection $\left[F(7,406)=64.647, p<.005, M S_{\mathrm{e}}=8.648\right]$, and false-alarm rate $\left[F(7,406)=15.12, p<.005, M S_{\mathrm{e}}=\right.$ 4.593]. The performance on the memory and monitoring tasks increased across sessions, but was not affected by the math payoff.

\section{Day 2}

Total points. There was a significant Day 2 payoff main effect $\left[F(1,56)=29.43, p<.005, M S_{\mathrm{e}}=42,182\right]$ and a session main effect $[F(3,168)=6.641, p<.005$, $\left.M S_{\mathrm{e}}=5,785\right]$. The groups who received MATH-15 payoff on Day 2 obtained more total points than did the groups who received a MATH-5 payoff.

The analysis of adjusted points showed a session main effect $\left[F(3,168)=8.9, p<.005, M S_{\mathrm{e}}=3,926\right]$ and, surprisingly, a Day 2 payoff main effect $[F(1,56)=4.91, p=$ $\left..03, M S_{\mathrm{e}}=22,469\right]$. This latter effect indicates that groups with higher math payoff obtained fewer adjusted total points than did the group with lower math payoff $(M=838$ for the MATH-5 group; $M=796$ for the MATH-15 group).

Individual tasks. Next, we will discuss the results for the math and remaining tasks.

Math task. The math score showed a significant Day 2 payoff main effect $\left[F(1,56)=89.26, p<.005, M S_{\mathrm{e}}=\right.$ $22,890]$, with the higher math payoff group obtaining a higher score than the lower math payoff group.

The adjusted math points showed a session main effect $\left[F(3,168)=5.14, p=.02, M S_{\mathrm{e}}=299\right]$ and a Day 2 payoff effect that approached significance $[F(1,56)=3.18, p=$ $\left..08, M S_{\mathrm{e}}=5,686\right](M=76$ for the MATH-5 group; $M=$ 93 for the MATH-15 group). However, the number of math responses showed only a session main effect $[F(3,168)=$ $\left.19.23, p<.005, M S_{\mathrm{e}}=5.9\right]$.

Remaining tasks. The memory task showed a significant Day 2 payoff main effect on task score (see Figure 7) $\left[F(1,56)=5.68, p<.021, M S_{\mathrm{e}}=14,247\right]$. The memory score was higher when the math score was low, and vice versa. Also, the session main effect was significant $\left[F(3,168)=7.38, p<.005, M S_{\mathrm{e}}=2,082\right]$. There was no significant Day 2 payoff main effect for the total number of memory responses (Figure 8) $[F(1,56)=1.41$, $p=.24]$, but the number of correct responses was significantly higher for the groups receiving 5 points for the math task (28.2) than it was for those receiving 15 points $(25.7)\left[F(1,56)=4.92, p<.031, M S_{\mathrm{e}}=71.26\right]$. 

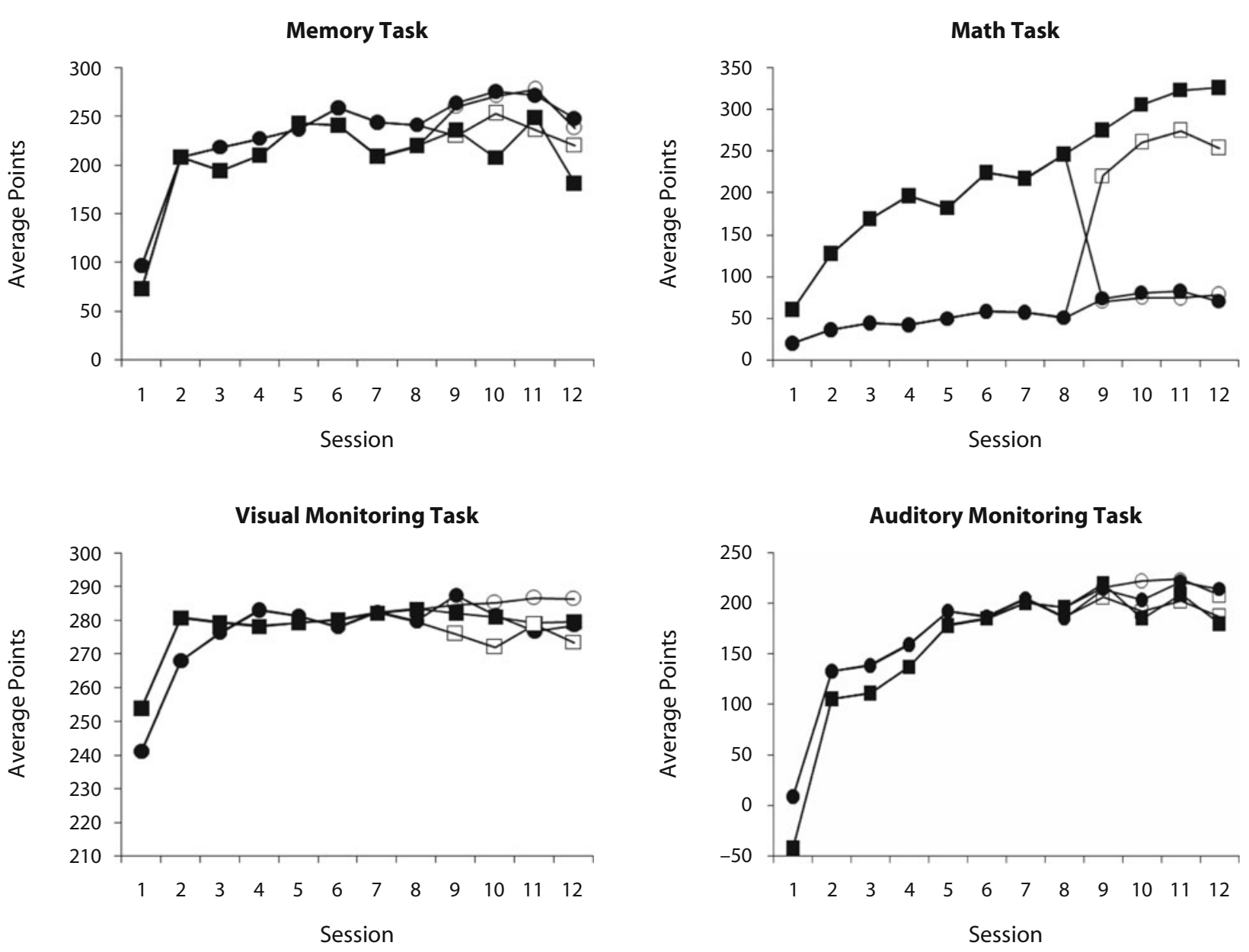

Figure 7. Individual task scores as a function of session and group for Experiment 2 . A math payoff of 15 is indicated by squares, and a math payoff of 5 is indicated by circles; filled symbols for the 2 nd day designate continued payoff, and unfilled symbols designate a changed payoff.

No term was significant for the visual task, suggesting that the $10-\mathrm{sec}$ window before the next response had to be made was sufficient to prevent any differences in priorities for the other tasks from having an impact. There was a significant Day 2 payoff main effect for auditory score $\left[F(1,56)=9.74, p=.003, M S_{\mathrm{e}}=1,952\right]$, with the lower math payoff group obtaining a higher auditory score $(M=$ 216 for the MATH-5 group; $M=198$ for the MATH-15 group). There was also a session main effect $(M \mathrm{~s}=214$, 201, 215, and 197 for Sessions 9-12, Sessions 9 and 11 obtained relatively lower auditory scores than did Session 10 and 12), and a session $\times$ Day 2 payoff interaction on task score $[F \mathrm{~s}(3,168)=7.96$ and 3.34, $p \mathrm{~s}<.005$ and $\left..021, M S_{\mathrm{e}}=593\right](M \mathrm{~s}=215,213,223$, and 211 for MATH-5, and $M \mathrm{~s}=213,188,206$, and 183 for MATH$15)$. There was a Day 2 payoff main effect for positive tone detection $\left[F(1,56)=9.36, p=.003, M S_{\mathrm{e}}=4.420\right](M=$ 23 for MATH-5, and $M=22$ for MATH-15) The session main effect and session $\times$ Day 2 payoff interaction for positive tone detection were also significant $[F \mathrm{~s}(3,168)=$ 8.41 and 3.14, $\left.p \mathrm{~s}<.05, M S_{\mathrm{e}}=1.398\right]$. Day 1 payoff had a main effect on false alarms $[F(1,56)=4.44, p=.04$,
$\left.M S_{\mathrm{e}}=0.375\right]$. All these results indicate that the auditory task performance was lower when participants paid more attention to the math task (in the higher math payoff group) than when they paid less attention to the math task (in the lower math payoff group).

Positive and negative incentives. As in Experiment 1, analyses of positive and negative incentives were performed for the explicit and implicit payoff differences.

Explicit effects. The positive incentive effect is the difference between the math-5/MATH-15 and math-5/ MATH-5 groups in Figure 8. When the payoff of the math task was increased on the 2nd day, participants performed 0.25 more math responses than did the group whose math task payoff remained the same as on the 1 st day. This small difference was nonsignificant $[F(1,28)<1.0]$.

The negative incentive effect is the difference in the number of math responses between the math-15/MATH-5 and the math-15/MATH-15 groups (see Figure 8). When the payoff of the math task was decreased, participants performed 6.68 fewer math responses than when the math payoff remained the same as on the 1st day $[F(1,28)=$ $\left.5.11, p=.032, M S_{\mathrm{e}}=262\right]$. This analysis confirms the 


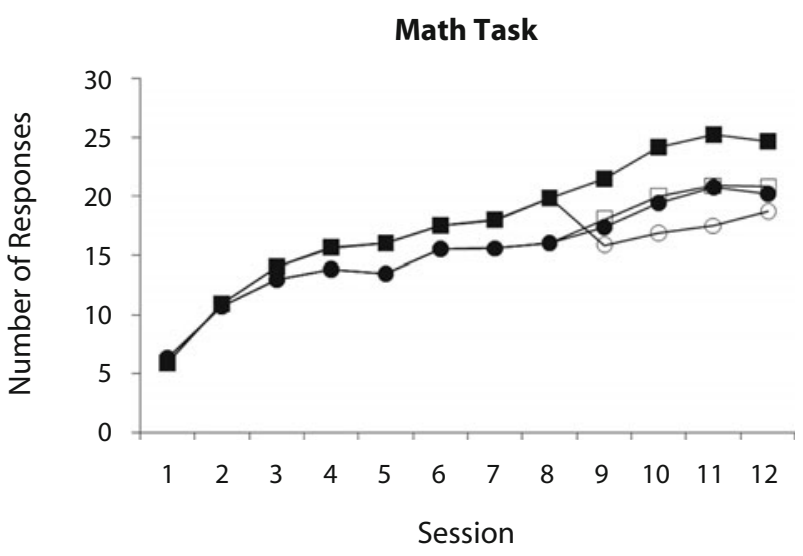

Figure 8. The number of math responses as a function of Sessions 1-8 (1st day) and Session 9-12 (2nd day) for Experiment 2. Math payoff of 15 is indicated by squares and a math payoff of 5 is indicated by circles; filled symbols for the 2 nd day designate continued payoff, and unfilled symbols designate a changed payoff.

finding of Experiment 1 that the negative incentive effect is more powerful than the positive incentive effect.

Implicit effects. The positive incentive effect is that the relative memory payoff is increased when the math payoff is decreased, which is the difference between the mean of math-15/MATH-15 and math-15/MATH-5, which was 1.83. This difference was not significant $[F(1,28)<1.0]$ and was due primarily to one individual in the math-15/ MATH-15 group. The positive incentive effect from the math task payoff change may have been obscured by a ceiling effect. In Experiment 2, the memory probe presentation rate was once every $10 \mathrm{sec}$, so a participant could easily reach the ceiling in both groups.

The negative incentive effect is that the relative memory payoff decreases when the math payoff is increased. The difference between the mean of the number of memory responses of math-5/MATH-5 and math-5/MATH-15 was 0.47 responses (see Figure 9). This difference in the number of memory responses was significant $[F(1,28)=4.31$, $\left.p=.047, M S_{\mathrm{e}}=1.515\right]$. On the whole, the data suggest that a negative incentive acts to reduce responding on the memory task, but that a positive incentive has little effect on performance.

\section{Discussion}

Although the payoff main effect for the number of math responses in the first eight sessions was not significant, the session $\times 1$ st day payoff interaction was significant. This interaction indicates that with practice, participants were sensitive to the payoff difference and performed the math task more when the payoff was high than when it was low. There were no significant payoff main effects or interactions for performance on the other tasks on the 1 st day.

For the 2nd day, the analysis did not indicate a significant main effect for payoff on the number of math responses or memory responses. Performance on the visual task was not affected by the payoff change. The auditory task showed that auditory task performance was worse when participants paid more attention to the math task (the higher math payoff group) than when they paid less attention to it (the lower math payoff group).

\section{GENERAL DISCUSSION}

When multitasking, people should be sensitive to the relative payoffs for the individual tasks. If the payoff for one is increased, the relative amount of effort devoted to that task should increase, whereas effort devoted to other tasks requiring the same information-processing resources should decrease. The present study examined the sensitivity of participants' multitasking performance to differences in initial payoff structure and payoff changes in the SYNWORK1 synthetic work environment. In both experiments, two groups performed the initial practice with payoff structures in which one task had higher payoff for one group than did the other. In the transfer portion of the experiments, either the same payoff continued to be in effect or the payoff for the task was changed to the alternative value. Thus, sensitivity to explicit payoffs and changes in payoffs for the one task could be evaluated, as could sensitivity to implicit payoffs and changes for the other tasks.

\section{Initial Payoff Structure}

In Experiment 1, the payoff for the memory task was 15 points for one group of participants and 5 points for another, but the math payoff was 10 points for both groups. Similarly, in Experiment 2, the math payoff was 15 points for one group and 5 points for the other, and the memory payoff was 10 points for both groups. In each experiment, the participants showed sensitivity to the explicit payoff difference: In Experiment 1, the memory task was performed increasingly more often across Sessions 1-8 by the participants receiving 15 points for each correct response than by those receiving 5 points. In Experi-

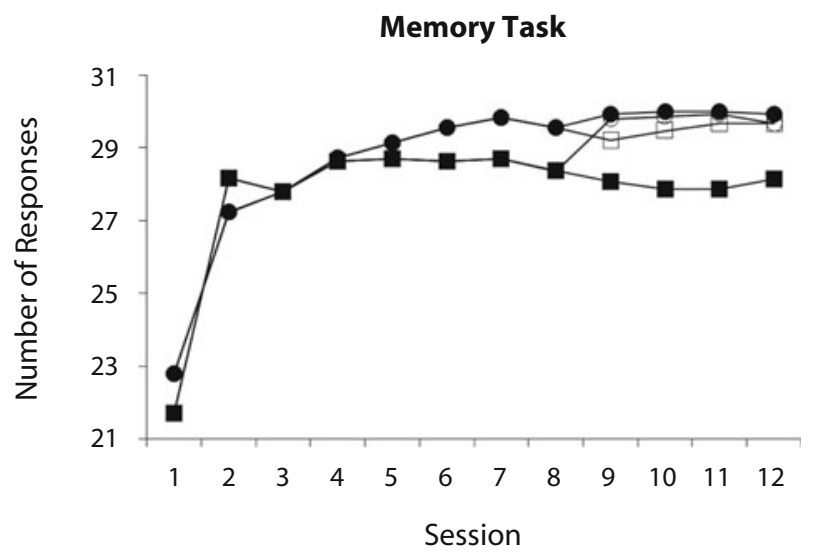

Figure 9. The number of memory responses as a function of Sessions 1-8 (1st day) and Sessions 9-12 (2nd day) for Experiment 2. Math payoff of 15 is indicated by squares, and a math payoff of 5 is indicated by circles; filled symbols for the 2 nd day designate continued payoff, and unfilled symbols designate a changed payoff. 
ment 2 , the math task was performed increasingly more often across Sessions $1-8$ by the participants receiving 15 points for each correct answer than by those receiving 5 points. In neither experiment, though, was there a significant decrease in performance of the cognitive task for which the payoff was not explicitly altered, nor was there a decrease in performance for the visual and auditory monitoring tasks. In other words, the increase in performance of the higher payoff group on the task for which the payoff differed was accomplished with little or no cost in performance of the other tasks. This finding suggests that the higher payoff was acting primarily to increase the effort devoted to performing the tasks as a whole and not to trading off performance of the lower and higher priority tasks.

When only the memory task payoff (Experiment 1) or only the math task payoff (Experiment 2) differed across conditions, the main effect of 1st-day payoff on the number of responses for that task in Sessions 1-8 was not significant, although its interaction with session was significant. In addition, the payoff manipulations in these experiments had no significant effect in Sessions 1-8 on the number of responses for the task in which the payoff was not explicitly varied. In comparison with our earlier study (Wang et al., 2007) in which a high payoff for the memory task was paired with a low payoff for the math task and vice versa, the tendency to alter the relative emphasis on performance of the respective tasks as a function of payoff differences is greater when the payoff for one task is high and the other is low than when payoff is explicitly varied for only one task. This outcome suggests that when training a person to perform complex tasks, explicit manipulation of the payoff relationships of the component tasks is important for the adoption of appropriate emphases. This conclusion is similar to that reached by Gopher et al. (1989).

\section{Transfer Payoff Structure}

Performance on the 2nd day for the participants who were transferred to the alternative payoff also showed sensitivity to the payoff structure, indicating that they altered the amount of effort devoted to the task for which the payoff was explicitly changed in accordance with that change. In both experiments, there was no significant effect of the 2nd-day payoff for the specific task to which the payoff difference applied (the memory task in Experiment 1 and the math task in Experiment 2). There was, however, a tendency in Experiment 1 for participants who had the higher memory payoff on the 2nd day to make more memory responses than those who had the lower payoff. Moreover, in Experiment 2, memory task performance showed an implicit effect of the 2nd-day payoff, with a higher point total obtained by the group for which the math payoff was low, due in part to a larger number of correct memory responses.

In Experiments 1 and 2, we measured positive and negative incentive effects for explicit and implicit payoff changes. The results showed that in a multitasking environment, an explicit change in the payoff for a task affected its performance. The decrease in performance resulting from the reduction of the payoff for one task (negative incentive) was larger than was the increase resulting from the increment of payoff for that task (positive incentive). Not only did the payoff change produce explicit effects, but it also tended to produce implicit effects on the performance of other tasks, particularly for performance of the memory task as a function of change in math-task payoff. This outcome is consistent with behavioral contrast results (see, e.g., Catania, 1963).

Comparison with the results of our previous experiment, in which the payoffs for the memory and math tasks were manipulated in opposition (Wang et al., 2007), shows that the changes in attention allocation are less when only the payoff on one task is changed than when the payoffs on two tasks are changed in opposite directions, and that an indirect increase in relative payoff of a task due to lowering the payoff on another task is less effective than a direct increase. A negative incentive is more effective than a positive incentive in producing explicit effects, indicating that the attention allocated to component tasks is affected differently when only one factor of an individual task is changed in either of two directions. Thus, when a negative incentive is applied in a multiple-task overloaded condition, the effect may be larger than would be expected.

Synthetic work environments allow control of cognitive load for multitasking situations similar to those encountered outside the laboratory and therefore have a history of use in the study of human performance (see, e.g., Alluisi, 1967; Morgan \& Alluisi, 1972). In the present study, participants showed sensitivity to the initial payoffs that were in effect and to changes in payoff that were introduced after practice. Our results indicate that the implicit manipulation of payoffs does not seem to be as effective as explicit manipulation, and that decreases in payoff produce greater changes in performance than do increases. When people are trained to perform in multiple-task environments, an explicit payoff schedule that emphasizes the relative importance of the respective tasks should be most effective. Furthermore, if one is interested in increasing the amount of effort devoted to a particular task, this can be accomplished by increasing the payoff for that task. If only the payoff for that task is increased, some performance gain may occur with little effect on performance of the other tasks.

Wickens (1980) proposed a multiple-resource model of attention that has come to be widely accepted in the human factors literature. The model distinguishes attentional resources on the basis of stages (perception, cognition, responding), modalities (visual, auditory), and codes (verbal; spatial; for responding, verbal-vocal and manual-spatial) of the tasks that are to be performed. In SYNWORK1, the memory-task stimuli are visual-verbal and the set of letters must be retained in working memory. For the math task, the input is also visual-verbal and requires computations that also need working memory. The stimuli for the visual and auditory tasks are nonverbal and use distinct modalities. However, the most salient aspect of SYNWORK1 is that responses for all tasks are made by positioning a pointer over an appropriate response box 
using a mouse and then clicking on the mouse. In this sense, all tasks have high visual-spatial and manualspatial demands, and the response mode necessarily imposes serial performance on the four tasks.

SYNWORK1 has proven to be a valuable research tool for investigating multiple-task performance (see, e.g., Salthouse, Hambrick, Lukas, \& Dell, 1996; Sit \& Fisk, 1999; Wang et al., 2007). However, it has several limitations, including that of allowing only the use of the same manualspatial response mode for all tasks, as was described previously. Another limitation is that the program is based on MS-DOS and therefore does not take advantage of the flexibility of contemporary computer systems. Although SYNWORK1 allows researchers to manipulate certain variables, such as payoffs for the individual tasks, presentation rates, and so on, it is limited in other respects. For example, the quadrants in which the tasks are performed are fixed. Also, all tasks are regularly paced, which does not allow the investigation of interruptions in performance of some tasks by the occurrence of an unpredictable stimulus for another task that requires immediate attention. Because SYNWORK1 and previous synthetic work tasks have proven to be valuable tools for investigating aspects of human performance, it is time for a new generation to be developed. The new generation should incorporate the distinctions identified as being important in multipleresource models of attention and in other work on dualtask performance (Wickens \& McCarley, 2008), as well as the full range of timing and task emphases that one could encounter in situations requiring multitasking.

\section{AUTHOR NOTE}

This research was supported in part by Grant W911NF-05-1-0153 from the Army Research Office. We thank Peter Urcuioli and E. J. Capaldi for providing useful information about the behavior contrast literature, and Kim Vu for helpful comments on the manuscript. We also appreciate the assistance of Lori Mansfield, Kelly Heaney, Jennifer Landwerlen, and Yi-Hsuen Shih in conducting the experiments. Correspondence should be sent to D. D. Wang, 1 UNF Dr., Jacksonville, FL 32224 (e-mail: dwang@unf.edu).

\section{REFERENCES}

AlLuisi, E. A. (1967). Methodology in the use of synthetic tasks to assess complex performance. Human Factors, 9, 375-384.

Catania, A. C. (1963). Concurrent performances: Reinforcement in- teraction and response independence. Journal of the Experimental Analysis of Behavior, 6, 253-263.

ElsMORE, T. F. (1994). SYNWORK1: A PC-based tool for assessment of performance in a simulated work environment. Behavior Research Methods, Instruments, \& Computers, 26, 421-426.

Gopher, D., Weil, M., \& Siegel, D. (1989). Practice under changing priorities: An approach to the training of complex skills. Acta Psychologica, 71, 147-177. doi:10.1016/0001-6918(89)90007-3

HERRNSTEIN, R. J. (1961). Relative and absolute strength of responses as a function of frequency of reinforcement. Journal of the Experimental Analysis of Behavior, 4, 267-272.

Herrnstein, R. J. (1990). Behavior, reinforcement and utility. Psychological Science, 4, 217-224. doi:10.1111/j.1467-9280.1990 .tb00203.X

LEVY, J., \& PASHLER, H. (2008). Task prioritization in multitasking during driving: Opportunity to abort a concurrent task does not insulate braking responses from dual-task slowing. Applied Cognitive Psychology, 22, 507-525. doi:10.1002/acp.1378

Logue, A. W., Forzano, L. B., \& Tobin, H. (1992). Independence of reinforcer amount and delay: The generalized matching law and self-control in humans. Learning \& Motivation, 23, 326-342. doi:10.1016/0023-9690(92)90012-B

Morgan, B. B., JR., \& Alluisi, E. A. (1972). Synthetic work: A methodology for assessment of human performance. Perceptual \& Motor Skills, 35, 835-845.

Proctor, R. W., WANG, D. Y., \& Pick, D. F. (1998). An empirical evaluation of the SYNWORK1 multiple-task work environment. Behavior Research Methods, Instruments, \& Computers, 30, 287-305.

Salthouse, T. A., Hambrick, D. Z., Lukas, K. E., \& Dell, T. C. (1996). Determinants of adult age differences on synthetic work performance. Journal of Experimental Psychology: Applied, 2, 305-329. doi:10.1037/1076-898X.2.4.305

Sit, R. A., \& FISK, A. D. (1999). Age-related performance in a multiple-task environment. Human Factors, 41, 26-34. doi:10 $.1518 / 001872099779577345$

Wang, D.-Y. D., Proctor, R. W., \& Pick, D. F. (2007). Acquisition and transfer of attention-allocation strategies in a multipletask work environment. Human Factors, 49, 995-1004. doi:10 $.1518 / 001872007$ X249866

WiCKENS, C. D. (1980). The structure of attentional resources. In R. S. Nickerson (Ed.), Attention and performance VIII (pp. 239-258). Hillsdale, NJ: Erlbaum

Wickens, C. D., \& McCarley, J. S. (2008). Applied attention theory Boca Raton, FL: CRC Press.

\section{NOTE}

1. An additional four sessions were conducted on Day 2, as in Wang et al.'s (2007) study, in which all participants changed to the opposite payoff from Sessions 9-12. These data are not reported since they yielded little of interest.

(Manuscript received November 28, 2008; revision accepted for publication March 24, 2009.) 